\title{
Variabilidade entre procedências de paricá Schizolobium parahyba var amazonicum (Huber ex Ducke) Barneby plantadas no município de Colares - Pará
}

\author{
Selma Toyoko OHASHIํㅜㄹ Jorge Alberto Gazel YARED², João Tomé de Farias NETO³
}

\section{RESUMO}

O estudo envolveu quatro procedências de paricá (Schizolobium parahyba var. amazonicum) plantadas no município de Colares-PA, seguindo um delineamento experimental em blocos ao acaso com cinco repetiçóes. As características estudadas foram: sobrevivência, altura da planta, diâmetro a 1,30m do solo (DAP), altura e diâmetro da copa, percentagem de plantas atacadas e tipo de casca. A avaliação foi efetuada aos três anos de idade. Foram encontradas diferenças entre procedências para a sobrevivência e crescimento em altura da planta e DAP, náo tendo sido encontradas diferenças para as características de altura da copa, diâmetro da copa, percentagem de plantas atacadas e tipo de casca. A procedência de maior sobrevivência foi Belterra que diferiu das demais ao nível de 5\% de significância. Para o crescimento em Altura da planta e DAP, as procedências Belterra, Ji-Paraná e Alta Floresta foram estatisticamente iguais entre si, diferindo de Brasiléia que apresentou o menor desenvolvimento. As procedência Belterra, Alta Floresta e Ji-Paraná podem ser recomendadas para o uso em programas de reflorestamento e sistemas agroflorestais para esta regiáo. O coeficiente de correlação de Spearman indicou alta associaçáo entre as variáveis de produção e a latitude, indicando que as procedências de latitudes mais baixas tendem a ter um maior desenvolvimento, entretanto, devido este estudo ter envolvido somente uma pequena amostra dentro da ampla área de distribuição da espécie, é aconselhável ampliar o trabalho de prospecção e coleta para melhor explorar a variabilidade no programa de melhoramento genético com a espécie.

PALAVRAS-CHAVES: variação genética; procedência, melhoramento genético, reflorestamento, Shizolobium amazonicum

\section{Variability among Schizolobium parahyba var. amazonicum (Huber ex Ducke) Barneby provenances planted in the municipality of Colares - PA}

\begin{abstract}
The study involved four three-year-old provenances of Schizolobium parahyba var. amazonicum planted in the municipality of Colares-Pará, following an experimental design of randomized blocks with five replications. The studied characteristics were: survival, plant height, diameter at $1.30 \mathrm{~m}$ from the ground (DAP), height and diameter of the tree crown, percentage of attacked plants and type of bark. Differences among origins for the survival and growth in height of plant and DAP had been found, however, no differences were found for crown height characteristics, crown diameter, percentage plants of attacked plants and bark type. The provenance with the highest survival was Belterra. For plant growth in height and DAP, the provenances Belterra, Ji-Paraná and Alta Floresta were statistically similar, differing from Brasiléia that presented the lowest development. The provenances Belterra, Alta Floresta and Ji-Paraná can be recommended for reforestation programs and agroforestry systems for this region. The Spearman correlation coefficient indicated a strong association among the production variables and the latitude, indicating that the provenances from lower latitudes tend to have a higher development. However, due to this study had involved only a small sample inside of the ample area of distribution of the species, it is advisable to extend the prospection and collecting activities for better explore the variability in the program of genetic improvement of the species.
\end{abstract}

KEYWORDS: genetic variation; provenance, genetic improvement, reforestation, Shizolobium amazonicum

\footnotetext{
1 Universidade Federal Rural da Amazônia, E-mail: selma.ohashi@ufra.edu.br

2 Empresa Brasileira de Pesquisa Agropecuária, Embrapa Amazônia Oriental, E-mail: jyared@amazon.com.br

${ }^{3}$ Empresa Brasileira de Pesquisa Agropecuária, Embrapa Amazônia Oriental, E-mail: tome@cpatu.embrapa.br
} 


\section{INTRODUÇÃO}

A variabilidade entre populaçóes sempre foi alvo de estudos em diferentes espécies visando conhecer e explorar esta variabilidade nos programas de melhoramento e conservação genética. Este fato se baseia na premissa que as populaçóes de diferentes origens geográficas apresentam diferenças genéticas que surgiram como resultado da adaptação às condiçôes ambientais locais em que vivem. Deste modo, é de se esperar que populaçôes de diferentes origens e diferentes condiçôes ecológicas apresentem diferenças adaptativas e morfológicas, sendo importante conhecer essas diferenças nos programas de plantio.

Os estudos de procedências em diferentes espécies têm demonstrado que existem diferenças entre elas em crescimento (Sotelo-Montes, et al., 2003; Hodge, et al., 2004, LopezUpton, et al., 2005), reprodução (Pilon, et al., 2003) produção de biomassa e componentes químicos (Liu, et al., 2002), capacidade de enraizamento de estacas (Puri \& Swamy, 1999) entre outros aspectos significando que a variabilidade entre populaçôes pode ser aproveitada de diferentes formas de acordo com a necessidade do sistema de produçáo.

A seleçấo de procedências tem contribuído sobremaneira para a obtençáo de materiais para multipropósitos, podendo-se citar a seleção de materiais genéticos resistentes a condiçôes áridas e pobres da África e Ásia (Tchoundjeu et al., 1999) para produção de forragem mais nutritivas para animais, (Arya, et al., 1995) para produção de lenha (Rawat \& Singh, 2000), para produção de frutos, lenha e madeira (Leakey \& Simons, 1998; Leakey, et al., 2002), entre outros com prioridades em diferentes regióes de acordo com a necessidade dos agricultores.

Desta maneira os ensaios de procedências são de grande valor por possibilitar conhecer e explorar o potencial das procedências em diferentes condiçôes ambientais e para diferentes finalidades.

A espécie Schizolobium parahyba var amazonicum (Huber ex Ducke) Barneby, conhecida vulgarmente como paricá, vem sendo muito utilizada nos sistemas de produção florestal e agroflorestal, pela qualidade de sua madeira para diferentes fins e pelo rápido crescimento em áreas alteradas, podendo fornecer bens e serviços, como prover sombreamento para os plantios de cacau e cupuaçu, ser o tutor vivo para a pimenta do reino, e ser componente de diferentes arranjos agroflorestais em pastagens e outros sistemas silviagrícolas.

Apesar da grande utilização desta espécie, estudos relacionados ao conhecimento da variabilidade genética sáo escassos sendo necessário ampliar este conhecimento para atender os sistemas de produçáo existentes no Estado do Pará, através da identificação e seleção de procedências produtivas, bem adaptadas a diferentes condiçôes ambientais e que possam atender a vários propósitos, como no caso de sistemas agroflorestais. Em vista do exposto, neste trabalho foram avaliadas quatro procedências utilizadas usualmente nos sistemas de plantio na regiáo, para caracteres de produção, com o objetivo de avaliar o comportamento produtivo e adaptativo destas procedências e seleçáo de materiais genéticos para os programas de reflorestamento e sistemas agroflorestais da região.

\section{MATERIAIS E MÉTODOS}

\section{LOCAL DE IMPLANTAÇÃO}

A pesquisa foi desenvolvida no município de Colares - PA localizado a $00^{\circ} 52^{\prime}$ Lat $S$ e $48^{\circ} 16^{\prime}$ Long W, caracterizando-se por apresentar precipitação de $2600 \mathrm{~mm}$ por ano com a maior concentraçáo de chuva de dezembro a junho e menor de julho a novembro. A temperatura média é de $26^{\circ} \mathrm{C}$ com mínima de 22 e máxima de $32^{\circ} \mathrm{C}$ (Dados de Belém-PA, INMET, 2004). $\mathrm{O}$ tipo de solo é latossolo amarelo distrófico textura média arenoso com relevo plano a levemente ondulado.

\section{PROCEDÊNCIAS UTILIZADAS}

Foram utilizadas quatro fontes de sementes localizadas na Amazônia Brasileira: Alta Floresta (Mato Grosso), Ji- Paraná (Rondônia), Brasiléia (Acre) e Belterra (Pará). As latitudes e longitudes e as condiçóes climáticas médias em termos de precipitaçâo e temperatura estão discriminadas na Tabela 1.

Essas procedências são utilizadas normalmente nos plantios comerciais no Estado do Pará. As sementes foram cedidas pelos Laboratórios de Sementes Florestais da AIMEX (Associação das Indústrias Madeireiras do Estado do Pará) e Embrapa Amazônia Oriental.

Tabela 1 - Latitude e longitude das procedências utilizadas no trabalho e do local de implantação dos ensaios experimentais com respectivos dados climáticos em termos de temperatura e precipitação.

\begin{tabular}{|c|c|c|c|c|c|c|}
\hline \multirow[b]{2}{*}{ Procedência } & \multirow[b]{2}{*}{ Latitude } & \multirow[b]{2}{*}{ Longitude } & \multirow{2}{*}{$\begin{array}{l}\text { Precipitação } \\
\text { total média } \\
(\mathrm{mm} / \mathrm{ano})^{1}\end{array}$} & \multicolumn{3}{|c|}{ Temperatura ${ }^{\circ} \mathrm{C}^{1}$} \\
\hline & & & & 点 & $\stackrel{\frac{\pi}{0}}{\frac{0}{0}}$ & 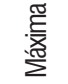 \\
\hline Brasiléia $(A C)^{2}$ & $11^{0} 00^{\prime} \mathrm{S}$ & $69^{\circ} 34^{\prime} \mathrm{W}$ & 1680 & 11,1 & 24,9 & 36,5 \\
\hline Ji-Paraná $(\mathrm{RO})^{3}$ & $09^{0} 58^{\prime} \mathrm{S}$ & $61^{0} 58^{\prime} \mathrm{W}$ & 2133 & 12,0 & 25,1 & 37,5 \\
\hline Alta Floresta (MT) ${ }^{4}$ & $09^{0} 53^{\prime} \mathrm{S}$ & $56^{0} 14^{\prime} \mathrm{W}$ & 2373 & 14,2 & 24 & 38,0 \\
\hline Belterra $(\mathrm{PA})^{5}$ & $02^{0} 38^{\prime} \mathrm{S}$ & $54^{0} 56^{\prime} \mathrm{W}$ & 1909 & 21,1 & 25,1 & 31,8 \\
\hline \multicolumn{7}{|c|}{$\begin{array}{l}\text { Dados INMET e Embrapa Monitoramento por Satélite. } \\
\text { 2Dados da Estação mais próxima Rio Branco Lat: } 9,58 \text { S e Long: } 67,29 \text { W. } \\
{ }^{2} \text { Dados da Estação mais próxima Porto Velho Lat: 8,76 S e Long: } 63,08 \mathrm{~W} \text {. } \\
{ }^{4} \text { Dados da Estação mais próxima Cidade Vera Lat: } 12,20 \text { S e Long: } 56,50 \mathrm{~W} \\
{ }^{5} \text { Dados da Estaçẫo mais próxima: Belterra : Lat: } 2,38 \text { S e Long: } 54,56 \mathrm{~W} .\end{array}$} \\
\hline \multicolumn{7}{|c|}{ DELINEAMENTO ESTATÍSTICO } \\
\hline
\end{tabular}

O delineamento estatístico adotado foi de blocos ao acaso com parcelas de 36 plantas e 25 plantas úteis em espaçamento de $4 \mathrm{x} 4 \mathrm{~m}$ e cinco repetiçóes. Para separar as parcelas e os blocos foram plantadas mudas de outras espécies florestais. 
A cobertura inicial da área era uma capoeira baixa formada após o abandono da área pelo desgaste ocasionado pelo uso agrícola.

O preparo da área foi realizado com roçagem e destocamento mecanizado. Em seguida foi feita a abertura mecanizada da cova com dimensóes de 30 × $85 \mathrm{~cm}$ em média e adubação por cova de 8 litros de 'cama de galinha' (composto orgânico a base de esterco de granja e serragem). A manutenção foi feita por roçagem mecânica três vezes por ano.

\section{COLETA DE DADOS}

A avaliação foi realizada, aos três anos, com base na mediçāo da altura da planta, diâmetro a 1,30m do solo (DAP), altura da copa, diâmetro da copa, característica de casca e ocorrência de ataque de praga.

A altura da planta e o tamanho da copa foram obtidos com o auxílio de uma régua de alumínio graduada em centímetros com $6 \mathrm{~m}$ de comprimento. As alturas superiores a esta medida foram obtidas por estimativa. O diâmetro da copa foi obtido pela média entre o maior e menor diâmetro da copa.

O DAP foi medido com fita diâmetrica. A ocorrência de pragas foi registrada com base nas perfuraçôes no fuste da planta, com contagem de plantas atacadas ou náo, sem considerar a intensidade do ataque.

As cascas foram classificadas como lisa, medianamente rugosa e rugosa. Esta classificação foi baseada em observaçôes no campo nos plantios em idades mais avançadas.

\section{ANÁLISE DE VARIÂNCIA}

A análise de variância foi realizada por local, não tendo sido efetuado a análise conjunta, uma vez que os experimentos possuem espaçamentos, idades e tratos culturais diferenciados, podendo estes fatores interferir nos resultados, por não haver padronização nos ensaios, considerando-se o seguinte modelo matemático:

$$
Y_{i j}=m+t_{i}+b_{j}+e_{i j}
$$

Onde: $Y_{i j}=$ valor observado da planta da procedência i dentro da parcela do bloco j;

$$
\begin{aligned}
& m=\text { média geral } \\
& t i=\text { efeito da procedência } \mathrm{i}(\mathrm{i}=1 \ldots 4) \\
& b j=\text { efeito do bloco } \mathrm{j}(\mathrm{j}=1 \ldots 5) \\
& e i j=\text { erro atribuído ao efeito da procedência i dentro do }
\end{aligned}
$$
bloco $\mathrm{j}$

As análises estatísticas foram feitas empregando-se o modelo fixo, com exceçẫo da média. A análise de variância para percentagem de sobrevivência e percentagem de plantas atacadas foi realizada utilizando-se os dados transformados pela seguinte expressão $Y=\operatorname{arcsen} \sqrt{\mathrm{X} / 100}$, de acordo Steel e Torrie, (1980).
Tabela 2 - Esquema de análise de variância utilizada para avaliar 0 efeito do bloco, da procedência e do erro experimental com os respectivos graus de liberdade (GL), esperanças do quadrado médio e teste $\mathrm{F}$.

\begin{tabular}{lcccc}
\hline $\begin{array}{l}\text { Fonte } \\
\text { Variação }\end{array}$ & $\mathrm{GL}$ & $\mathrm{QM}$ & $\mathrm{E}(\mathrm{QM})$ & $\mathrm{F}$ \\
\hline Bloco & $\mathrm{r}-1$ & & \\
\hline Procedência & $\mathrm{t}-1$ & $\mathrm{Q}_{2}$ & $\boldsymbol{v}_{e}^{2}+r v_{p}^{2}$ & $\mathrm{Q}_{2} / \mathrm{Q}_{1}$ \\
\hline Erro & $(\mathrm{t}-1)(\mathrm{r}-1)$ & $\mathrm{Q}_{1}$ & $v_{e}^{2}$ \\
\hline Total & $\mathrm{t}$.r-1 & & \\
\hline
\end{tabular}

O esquema de análise de variância encontra-se na Tabela 2.

Para verificar a existência de variabilidade entre procedências, empregou-se o teste $\mathrm{F}$ a $5 \%$ de probabilidade. Para caracteres cujo teste $\mathrm{F}$ foi significativo empregou-se o teste de Duncan de comparação das médias das procedências por discriminar com mais facilidade os tratamentos

A estimativa dos componentes de variância seguiu o método utilizado por Yared (1983). A variância genética entre procedências e a variância ambiental foram calculadas a partir da esperança do quadrado médio obtidos na análise de variância.

O coeficiente de correlação de Spearman (Steel e Torrie, 1980) foi utilizado para verificar a associação entre a latitude e os caracteres avaliados: percentagem de sobrevivência, altura, DAP e percentagem de plantas atacadas. O emprego deste tipo de correlação é a mais indicada neste estudo pelo fato de usar a classificação das variáveis estudadas para verificar a relação existente entre elas.

Para comprovar a significância de um valor obtido de $r$ sob a hipótese de nulidade (as variáveis não estão associadas) fez-se o teste de distribuição t de Student.

\section{RESULTADOS E DISCUSSÃO}

Avaliação dos caracteres sobrevivência; altura, diâmetro a 1,30m do solo (DAP), diâmetro da copa e altura da copa.

$\mathrm{Na}$ Tabela 3 são apresentados os principais valores $\mathrm{da}$ análise de variância para os caracteres percentagem de sobrevivência, altura da planta, DAP, altura da copa e diâmetro da copa; o coeficiente de variação ambiental $\left(\mathrm{CV}_{\mathrm{e}} \%\right)$, o coeficiente de variação genético $\left(\mathrm{CV}_{\mathrm{g}} \%\right)$; a média geral do caracter; e o valor de F calculado e a significância.

Tabela 3. Resumo da análise de variância para sobrevivência, altura da planta, DAP, diâmetro da copa e altura da copa para um ensaio de procedências de Schizolobium parahyba var 
Tabela 3 - Resumo da análise de variância para os caracteres: sobrevivência, altura da planta, diâmetro a 1,30 m do solo, diâmetro da copa e altura da copa para um ensaio de procedências de Schizolobium parahyba var amazonicum (Huber ex Ducke) Barneby aos três anos de idade plantado no município de Colares - PA.

\begin{tabular}{|c|c|c|c|c|c|c|}
\hline \multirow[b]{2}{*}{$\begin{array}{l}\text { Fonte de } \\
\text { variação }\end{array}$} & \multirow[b]{2}{*}{ GL } & \multicolumn{5}{|c|}{ QM } \\
\hline & & Sobrevivência & Altura & DAP & $\begin{array}{l}\text { Diâmetro } \\
\text { da copa }\end{array}$ & $\begin{array}{c}\text { Altura da } \\
\text { copa }\end{array}$ \\
\hline Bloco & 4 & 55,28 & 0,52 & 0,15 & 0,03 & 0,11 \\
\hline Procedência & 3 & $625,60^{*}$ & 2,67 * & $2,45^{\star}$ & $0,02 \mathrm{~ns}$ & $0,16 \mathrm{~ns}$ \\
\hline Erro & 12 & 110,43 & 0,80 & 0,62 & 00,02 & 0,09 \\
\hline $\mathrm{CV}_{\mathrm{e}} \%$ & & 15,81 & 12,31 & 7,80 & 5,69 & 17,24 \\
\hline $\mathrm{CV}_{\mathrm{g}} \%$ & & 12,14 & 4,93 & 7,76 & & \\
\hline Média Geral & & 83,61 & 6,84 & 10,07 & 2,44 & 1,74 \\
\hline $\begin{array}{l}\text { F calculado } \\
\text { Procedência }\end{array}$ & & 5,67 & 3,75 & 3,95 & 1,00 & 1,78 \\
\hline
\end{tabular}

Valor de $\mathrm{F}$ tabelado a $5 \%$ de probabilidade $=3,49$

amazonicum (Huber ex Ducke) Barneby aos três anos de idade plantado no município de Colares - PA.

Diferenças em sobrevivência, altura da planta e DAP foram encontradas entre as procedências detectadas pela análise de variância cujo valor de $\mathrm{F}$ acusou diferenças estatísticas significativas ao nível de $5 \%$ de probabilidade, não tendo sido verificadas diferenças estatísticas significativas para altura da copa e diâmetro da copa.

Os valores dos coeficientes de variação ambiental $\left(\mathrm{CV}_{\mathrm{e}} \%\right)$ obtidos são considerados medianos, segundo os padróes apresentados por Garcia (1989) baseados em Pinus e Eucalyptus, indicando boa precisão experimental, podendo-se portanto ter confiabilidade nos resultados obtidos.

A relação entre o coeficiente de variação genética e o coeficiente de variação experimental é um dado importante para o melhorista. Em ensaios de progênie quando esta relação atinge o valor 1,0 ou superior indica uma situaçáo favorável à seleção (Vencovsky \& Barriga, 1992) devido ao controle eficiente da precisão experimental e pelas diferenças encontradas entre os materiais genéticos utilizados. Se esta relação for considerada para os coeficientes de variação genético e ambiental do ensaio de procedência, a seguinte situação vai ocorrer: para sobrevivência e DAP, os valores da relação $\mathrm{CV}_{\mathrm{g}}$ ! $\mathrm{CV}_{\mathrm{e}}$ foram 0,77 e 0,99 , respectivamente, significando que a seleção de procedências para estas características terá maior eficiência do que para a variável altura total que apresentou o valor desta relaçáo de 0,40 .

A altura da copa e o diâmetro da copa não apresentaram diferenças estatísticas entre as procedências estudadas. As plantas em sua maioria apresentavam copas sem ramificaçóes laterais e folhas grandes, características comuns na espécie no estágio inicial de desenvolvimento. A avaliação desta característica em estágios posteriores deve ser efetuada uma vez que esses caracteres são importantes para os sistemas agroflorestais.
No levantamento de plantas atacadas, verificou-se pequena percentagem de plantas com o fuste perfurado ou com marcas de ocorrência de ataque em todas as procedências testadas, náo tendo a análise de variância detectado diferenças entre si. Os resultados médios de percentagem de ataque foram: 0,7 \% para Brasiléia, 2,8 \% para Belterra, 5,6 \% para Alta Floresta e 6,7\% para Ji-Paraná. Porém estes resultados podem ter sido mascarados pela mortalidade das árvores, não podendo ser feita inferência da resistência da procedência quanto ao valor encontrado, uma vez que a incidência de pragas pode ter influenciado na mortalidade de plantas no ensaio.

Diferença no tipo de casca não foi encontrada entre as procedências analisadas. Considerando que em observaçôes realizadas em plantios comerciais em idades mais avançadas aparecem manifestações desse fenômeno, mesmo em baixa freqüência, é necessário considerar este caráter nas avaliaçóes posteriores.

Variabilidade para tipo de casca tem sido encontrada em Eucalyptus urophylla apresentando três tipos de casca: casca lisa, meia casca e casca rugosa; porém, nessa espécie, o fenótipo casca lisa apresenta baixa freqüência e meia casca e casca rugosa maior ocorrência nas populações (Scanavaca Júnior \& Garcia, 2003; Luz, 1997)

Para as variáveis que apresentaram diferenças detectadas pelo teste F, sendo elas percentagem de sobrevivência, altura total da planta e DAP, as médias e o teste de Duncan de comparação de médias a 5\% de probabilidade estâo apresentados na Tabela 4. Os resultados encontrados de percentagem de sobrevivência, altura total da planta e DAP indicam que existe variabilidade genética entre as procedências estudadas. Estes resultados podem ser explicados pelo fato de que as populaçóes desenvolveram-se em ambientes distintos e, portanto, com adaptabilidade à condição ambiental local.

Tabela 4 - Dados médios por procedência para sobrevivência, altura total da planta e diâmetro a 1,30m do solo (DAP) para procedências de Schizolobium parahyba var. amazonicum (Huber ex Ducke) Barneby aos três anos de idade plantadas no município de Colares-PA.

\begin{tabular}{lccc}
\hline Procedência & $\begin{array}{c}\text { Sobrevivência } \\
(\%)\end{array}$ & $\begin{array}{c}\text { Caracteres } \\
\text { Altura }(\mathrm{m})\end{array}$ & DAP $(\mathrm{cm})$ \\
\hline Belterra & $93,89 \mathrm{a}$ & $7,38 \mathrm{a}$ & $10,34 \mathrm{a}$ \\
Jiparaná & $87,78 \mathrm{bc}$ & $7,07 \mathrm{a}$ & $10,44 \mathrm{a}$ \\
Alta Floresta & $82,78 \mathrm{bc}$ & $7,15 \mathrm{a}$ & $10,50 \mathrm{a}$ \\
Brasiléia & $70,00 \mathrm{~b}$ & $5,76 \mathrm{~b}$ & $9,03 \mathrm{~b}$ \\
Média Geral & 83,61 & 6,84 & 10,07 \\
\hline
\end{tabular}

Para a percentagem de sobrevivência, o maior valor encontrado foi na procedência Belterra seguida de Ji-Paraná, Alta Floresta e Brasiléia. Para Altura e DAP, os maiores valores foram nas procedências Belterra, Alta Floresta e Ji-Paraná com igualdade estatística entre si diferenciando-se de Brasiléia. Desta maneira, devido à procedência Brasiléia ter apresentado menor sobrevivência e menor desenvolvimento (Tabela 4), 
nesta fase inicial, pode-se considerar que esta procedência é de menor adaptabilidade ao local.

\section{CORRELAÇÃO ENTRE AS VARIÁVEIS DE PRODUÇÃO E A LATITUDE}

Para verificar se a latitude está associada às características de sobrevivência e produçáo, calculou-se o coeficiente de correlação por postos de Spearman (rs) $(\mathrm{Ho}=\mathrm{rs}=0$ e H1= $\mathrm{rs} \neq 0$ ), relacionando os resultados às diferenças ambientais que influenciaram a evolução da espécie em cada latitude. Foi encontrada correlação significativa somente entre a latitude e a variável altura (Tabela 5) e correlação não significativa para a sobrevivência e DAP, ou seja, a variação em latitude teve uma associaçáo inversa com variável altura da planta uma vez que a correlaçáo foi negativa, ou seja, quanto maior for a latitude menor será o crescimento em altura nas condiçôes de Colares.

Para uma melhor avaliação desta associação é necessário o uso de um maior número de procedências e um maior controle ambiental na experimentaçáo, para que sejam feitas inferências mais conclusivas quanto a este aspecto, porém os

Tabela 5 - Coeficientes de correlação de Spearman entre a latitude e os caracteres percentagem de sobrevivência, altura da planta, diâmetro a $1,30 \mathrm{~m}$ do solo (DAP) e plantas atacadas de Schizolobium parahyba var. amazonicum (Huber ex Ducke) Barneby) aos três anos de idade no município de Colares - PA.

\begin{tabular}{lcc}
\hline Associação entre & $\begin{array}{c}\text { Coeficiente de correlação } \\
r_{s}\end{array}$ & Valor de $t$ \\
\hline Latitude x sobrevivência & $-0,80$ & 1,89 \\
Latitude x altura & -1 & \\
Latitude x DAP & $-0,40$ & 0,62 \\
\hline
\end{tabular}

resultados encontrados indicam já existir uma tendência de associação entre estes dados.

Associação deste tipo tem sido encontrada ( $\mathrm{Li}$, et al., 1998) em estudos com Arabidopsis thaliana em que a taxa de crescimento tem valores menores para as procedências de maiores latitudes e em termos gerais o que se verifica é que a latitude tem uma grande influência no padrão de distribuição das plantas (Santamaria et al., 2003), refletindo no padráo de riqueza de espécies (Qian, 1999), na fenologia (Olsson \& Agren, 2002), crescimento (Sawada et al., 1994), reprodução sexual (Winn \& Gross, 1993), forma da folha (Hebert, 2003), entre outras. Desta maneira os resultados encontrados reforçam os dados já encontrados na literatura. A sobrevivência e o crescimento do paricá tendem a apresentar variação seguindo o gradiente latitudinal e estas diferenças são importantes na seleçâo de materiais genéticos mais produtivos.

A seleçáo de procedências difere de acordo com o local e finalidade desejada. Podem ser selecionadas procedências para maior eficiência nutricional e resistência à geada na regiáo sul do Brasil (Caldeira et al., 2002), para crescimento, sobrevivência e tolerância à seca na regiāo noroeste de Minas Gerais (Marques Júnior, et al., 1996), para crescimento, produçấo volumétrica e qualidade da madeira (Sampaio, et al., 2002), entre outros aspectos,

O enfoque do programa de melhoramento desta maneira deve atender às necessidades dos locais, às peculiaridades do sistema de produçáo adotado e às necessidades dos agricultores.

O paricá tem sido utilizado nos sistemas agroflorestais pelos agricultores da regiáo para o sombreamento do cacau (Theobroma cacao) (Barros, et al., 2002) e do cupuaçu (Theobroma grandiflorum) (Ohashi, et al., 2004), e nos reflorestamentos tem sido adotado no sistema taungya (Ohashi, 2002) e como tutor vivo da pimenta-do-reino em alguns casos. Nestes sistemas, a qualidade de copa e a casca são de grande importância, devendo ser avaliados posteriormente no ensaio.

Os resultados encontrados reforçam a importância do estudo das características de produçáo para conhecer a adaptabilidade aos locais de plantio. Os resultados mais favoráveis em sobrevivência e crescimento para a procedência de Belterra podem ser atribuídos ao componente genético da população e/ou pela similaridade climática e proximidade geográfica entre o ambiente onde as sementes foram coletadas e o local onde foi implantado o ensaio. Belterra localiza-se a $2^{\circ}$ $63^{\prime}$ lat $S$ e $54^{\circ} 94^{\prime}$ long. W, e o local de experimentaçáo está a $0^{\circ} 56^{\prime}$ lat $S$ e $48^{\circ} 16^{\prime}$ long. W, e estes locais são também os que apresentam condiçôes climáticas mais semelhantes.

As procedências Alta Floresta e Ji-Paraná, apesar de terem apresentado uma sobrevivência menor em relaçáo a Belterra, apresentaram um bom crescimento podendo ser recomendadas para uso nas condiçôes ambientais de Colares, porém avaliaçôes posteriores são necessárias uma vez que a presente avaliação ocorreu ainda no estágio juvenil.

Para a procedência de Brasiléia que apresentou o pior desempenho em todas as variáveis analisadas, a distância geográfica e as condiçôes climáticas são também mais contrastantes em relação ao ambiente do local de experimentação, podendo este fato ter influenciado na adaptação à condição ambiental local do experimento.

A existência de variabilidade genética torna possível a seleção de materiais genéticos mais adaptados a condições ambientais específicas e para diferentes sistemas de produção. Para recuperação de áreas alteradas pelo uso do reflorestamento e sistemas agroflorestais, um trabalho mais amplo de prospecção e coleta é necessário para ampliar a base genética e obtenção de variabilidade para diferentes caracteres úteis ao melhoramento. 


\section{CONCLUSÕES}

Os resultados apresentados e discutidos neste trabalho permitem as seguintes conclusôes:

As procedências testadas apresentam diferenças em adaptabilidade detectada pela sobrevivência diferencial, significando que o uso de materiais genéticos mais adaptados proporcionará ganhos por conferir menor perda no plantio e, conseqüentemente, menor gasto de tempo e mão-de-obra. Para o município de Colares, dos materiais genéticos testados, as procedências Belterra, Alta Floresta e Ji-Paraná foram as de melhor comportamento podendo ser recomendadas para plantio para as condiçôes deste local nesta fase de desenvolvimento;

Diferenças para os caracteres de copa e casca não foram encontradas entre as procedências testadas na idade avaliada, porém há necessidade de acompanhamento em idade mais avançada para inferir sobre a variabilidade para estes caracteres, os quais são de grande importância para os programas de reflorestamento e sistemas agroflorestais.

\section{BIBLIOGRAFIA CITADA}

Arya, S.; Bisht, R.P.; Tomar, R.; Toky, O.; Pharris, P.J.C. 1995. Genetic variation in minerals, crude protein and structural carbohydrates of foliage in provenances of yong plants of Prosopis cineraria (L.) Druce in India. Agroforestry Systems, 29: 1-7.

Barros, A.V.; Ohashi, S.T.; Silva, P.T.E.; Kato, O.R.; Brienza Júnior, S.; Yared, J.A.G. 2002. Componentes e Arranjos de Espécies em Sistemas Agroflorestais Praticados por Agricultores NipoBrasileiros no Município de Tomé-Açu - Pará. Congresso Brasileiro de Sistemas Agroflorestais, 4. Anais... lhéus-BA, Out/2002. Cd-rom

Caldeira, M.V.W.; Rondon Neto, R.M.; Schumacher, M.V. 2002. Avaliação da eficiência nutricional de três procedências australianas de acácia negra (Acácia mearnsii Wild.). Revista Árvore, 26: 615-620.

Garcia, C.H. 1989. Tabelas para classificação do coeficiente de variação. (Circular Técnica). Piracicaba: IPEF, 1989. n. $171,11 \mathrm{pp}$.

Hodge, G.R.; Dvorak, W.S. 2004. The CAMCORE international provenance/progeny trials of Gmelina arborea: genetic parameters and potential gain. New Forests, 28: 147 - 166.

Instituto Nacional De Meteorologia. 2004. (http://www.inmet. gov.br/climatologia). Abril de 2004.

Lopez-Upton, J.; Donahue, J.K.; Plascencia-Escalante, F.O; Ramirez-Herrera, C. 2005. Provenance variation in growth characters of four subtropical pine species planted in Mexico, New Forests, 29: 1-13.

Kassen, R.E. Bell, G. 2000. The ecology and genetics of fitness in Chlamydomonas. X. The relationship between genetic correlation and genetic distance. Evolution, Lawrence, 54: 425-432.
Leakey, R.R.B.; Atangana, A.R.; Kengni, E.; Waruhiu, A.N.; Usoro, C.; Anegbeh, P.O.;

Leakey, R.R.B.; Simons, A.J. 1998. The domestication and commercialization of indigenous trees in agroforestry for the alleviation of poverty. Agroforestry Sistemas. 38: 165-176.

Li, B.; Suzuki, J.; Hara, T. 1998. Latitudinal variation in plant size and relative growth rate in Arabidopsis thaliana, Oecologia, 115: 293-301.

Liu, Z.; Zhou, G.; Xu, S.; Wu, J.; Yin, Y. 2002. Provenance variation in camptothecin concentrations of Camptotheca acuminata grown in China. New Forests, 24: 215-224.

Luz, H.F. 1997. Comparação de progênies de populações naturais e raças locais de Eucalyptus urophylla S.T.Blake. Dissertação de Mestrado- ESALQ - Universidade de São Paulo,SP, Piracicaba, $191 \mathrm{pp}$.

Mafongoya, P.L.; Chintu, R.; Chirwa, T.S. ; Matibini, J.; Chikale, S. 2003. Tephrosia species and provenances for improved fallows in southern Africa. Agroforestry Systems, 59: 279288.

Marques Júnior, O.G.; Andrade, H.B.; Ramalho, M.A. 1996. Avaliação de procedências de Eucalyptus cloeziana F. Muell.E. estimação de parâmetros genéticos e fenotípicos na região noroeste do Estado de Minas Gerais. (http://www.dcf. ufla.br/cerne/rev-v2n1-1996.htm). Acessado em Jan/2004.

Mcdonald, M.A.; Healey, J.R.; Jones, M. 2000. Variations and phenotypic correlation of growth attributes of Calliandra calothyrsus in the Blue Mountains of Jamaica. Agroforestry Sistems, 50: 293-314.

Ohashi, S.T.; Batista, T.F.C.; Barros, A.V.; Yared, J.A.G, Terezo, E. 2002. Influência da Aplicação de Calcário e Uso de Caupi (Vigna unguiculata) no Desenvolvimento de Espécies Florestais, na Fase Inicial de Implantação no Município de Aurora do Pará - PA. Congresso Brasileiro de Sistemas Agroflorestais, 4. Anais... Ilhéus-BA. Cd-rom.

Ohashi, S.T.; Silva,P. T.E.; Yared, J.A.G.; Brienza Júnior, S.; Kato, O.R.; Takamatsu, J.A. 2004. Sistema Silviagrícola Multiestratificado : In - Comportamento Produtivo De Paricá (Schizolobium amazonicum Huber), Açaí (Euterpe oleracea Mart) E Cupuaçu (Theobroma grandiflorum (Willd.) ex Spr.) K. Schum.) no Município de Tomé-Açu (PA). Congresso Brasileiro de Sistemas Agroflorestais, 4. Anais... Curitiba- PR. Cd-rom.

Olsson, K.; Agren, J. 2002. Latitudinal population differentiation in phenology, life history and flower morphology in the perennial herb Lythrum salicaria. Journal of Evolutionary Biology. 15: 983-996.

Pilon, J.; Santamaría, L.; Hootsmans, M.; Vierssen, W. Van. 2003. Latitudinal variation in life-cycle characteristics of Potamogeton pectinatus L.: vegetative growth and asexual reproduction, Plant Ecology, 165: 247-262.

Puri, S.; Swamy, S.L. 1999. Geographical variation in rooting ability of stem cuttings of Azadirachta indica and Dalbergia sissoo, Genetic Resources and Crop Evolution, 46: 29-36. 
Qian, H. 1999. Spatial pattern of vascular plant diversity in North Amarica North of Mexico and its floristic relationship with Eurasia. Annals of Botany, 83: 271-283.

Rawat, J.S.; Singh, T.P. 2000. Seedling indices of four tree species in nursery and their correlations with field growth in Tamil Nadu, India. Agroforestry Systems, 49: 289-300.

Scanavaca Júnior, L.; Garcia, J.N. 2003. Potential of genetic improvement I Eucalyptus urophylla from the Island Flores. Scientia Forestalis, 64: 23-32.

Sampaio, P.T.B.; Resende, M.D.V.; Araújo, A.J. 2002. Estimativas de parâmetros genéticos e métodos de seleçáo para o melhoramento genético de Pinus oocarpa Schiede. Pesquisa Agropecuária Brasileira, 37: 625-636.

Santamaria, L.; Figuerola, J.; Pilon. J.J. 2003. Plant performance across latitude: The role of plasticity and local adaptation in an plant. Ecology, 81: 2154-2161.

Sawada, S.; Nakajima,Y.; Tsukuda, M. et al.. 1994. Ecotype differentiation of dry matter production proceses in relation to survivorship and reproductive potential in Plantago asiática populations along climatic gradients. Funtional Ecology, 8: 400-409.

Sotelo Montes, C.; Vidaurre, H.; Weber, J. 2003. Variation in stem-growth and branch-wood traits among provenances of Calycophyllum spruceanum Benth. from the Peruvian Amazon, New Forests, 26: 1-16.
Steel, R.G.D.; Torrie, J.H. 1980. Principles and procedures of Statistics A biometrical Approach. 2a ed. McGraw-Hill Book Company. New York, 633 Pp.

Tchoundjeu, Z.; Weber, J.; Guarino, L. 1999. Germoplasm collections of endangerede agroforestry tree species: the case of Prosopis africana in the semi-arid lowlands of West Africa. Agroforestry Systems, 39: 91-100.

Vencovsky, R.; Barriga, P. 1992. Genética Biometrica no melhoramento. Ribeirão Preto, SBG, 486 pp.

Weber, J.C.; Montes, C.S.; Vidaurre, H.; Dawson, I.K. ; Simons, A.J. 2001. Participatory domestication of agroforestry trees: an example from the Peruvian Amazon. Development in Practice, 11: 425-433.

Winn, A.A.; Gross, K.L. 1993. Latitudinal variation in seed weight and flower number in Prunella vulgaris. Oecologia. 93: 55-62.

Yared, J.A.G. 1983. Comportamento e variabilidade de procedências de Cordia alliodora (Ruiz e Pav.) Oken no planalto do Tapajós - Belterra - PA. Dissertação de Mestrado - ESALQ - Universidade de São Paulo. Piracicaba, 109 pp.

Recebido em: 16/05/2008

Aceito em: 04/05/2009 
